\title{
Dextran sulphate induced arthritis in rabbits
}

\author{
J. STEINBERG, ${ }^{1}$ C. B. SLEDGE, ${ }^{2}$ J. NOBLE, ${ }^{2}$ AND H. G. GILliES ${ }^{3}$
}

From the Orthopaedic Research Laboratory, Robert B. Brigham Hospital, Departments of ${ }^{1}$ Medicine, Peter Bent Brigham Hospital, Orthopaedic Surgery, ${ }^{2}$ Robert B. Brigham Hospital, and ${ }^{3}$ West Roxbury Veterans Administration Medical Center, Harvard Medical School, Boston, Massachusetts 02120, USA.

SUMMARY Persistent effects of a short course of intra-articular dextran sulphate, dextran, or chondroitin sulphate were examined in rabbit knees. Only dextran sulphate produced gross arthritis, associated with high synovial acid phosphatase and beta-glucuronidase activities. Synovial degradative capacity in synovium-cartilage cocultures was increased 2 -fold by dextran sulphate and $1 \cdot 5$-fold by chondroitin sulphate treatments. Stimulation of cartilage breakdown in vitro paralleled the content of synovial marker enzyme at death of the animal, but the 2 responses could be dissociated.

Destruction of articular cartilage in various human arthropathies may proceed via a final common effector pathway ${ }^{1}$ mediated through the action of degradative enzymes. ${ }^{2.3}$ Whatever the primary event, subsequent breakdown and abortive repair ${ }^{4-7}$ result from tissue interactions in which both synovial and cartilage elements participate. ${ }^{8,9}$

It has been suggested that proteoglycans released by the initial injury from normally sequestered sites in cartilage matrix may promote or sustain the degradative response, and that the arthritogenic potential of these complex polysaccharides may be a function of their large size or their high negative charge. Polysaccharide macromolecules, depending on their size and degree of sulphation, have been shown to release lysosomal enzymes in vitro from macrophages ${ }^{10}$ and embryonic cartilage. ${ }^{11}$ Intraarticular injection of carrageenin in rabbit knees has been used to produce an inflammatory arthritis with high synovial enzyme activity and loss of cartilage proteoglycan. ${ }^{12-15}$ Florid synovitis and destructive osteoarthritis were similarly produced in rabbit knees by repeated intra-articular injection of dextran sulphate. ${ }^{16}$ This could not be reproduced by injection with uncharged high molecular weight dextran, or with the smaller sulphated polysaccharides, heparin and chondroitin sulphate. Because even a brief course of dextran sulphate appeared sufficient to induce this response, we chose to compare the effects persisting in synovium 2 months

Accepted for publication 31 July 1979

Correspondence to James Steinberg, MD, Orthopaedic Research Laboratory, Robert B. Brigham Hospital, 125 Parker Hill Avenue, Boston, Massachusetts 02120, USA. after exposure to dextran, dextran sulphate, or chondroitin sulphate as representative high molecular weight and/or highly charged polysaccarides.

\section{Materials and methods}

Dextran sulphate (type 500-S, average mol wt $5 \times 10^{5}$, sodium salt), dextran (average mol wt $5 \times 10^{5}$ ) and chondroitin sulphate (mixed isomers ex-whale and shark cartilage, grade III) were prepared as aqueous solutions $(50 \mathrm{mg} / \mathrm{ml})$ and sterilised by passage through a Millipore filter $(0.45 \mu \mathrm{m})$. Adult New Zealand white rabbits received a total of 3 intra-articular injections $(25 \mathrm{mg}$ per $0.5 \mathrm{ml}$ ) of the same test substance at 2-day intervals. In all animals dextran sulphate was injected through the patellar ligament into one knee and neutral dextran, or chondroitin sulphate, or an equal volume of $0.9 \%$ saline was injected into the other. Animals were killed 8 weeks after the first injection. Intact knees were removed aseptically, dissected free of adherent tissue, and the synovial lining excised from above. The synovial membrane was stripped of adherent capsular and extraarticular tissues, placed in a Petri plate moistened with Gey's balanced salt solution, and finely minced with a scalpel and dissecting scissors. Portions were immediately removed and chilled on ice for subsequent enzyme assay. In some experiments the balance of this tissue was used to assay proteoglycan degradative capacity as outlined below.

Chilled synovia were blotted, weighed, and homogenised for $1 \frac{1}{2} \mathrm{~min}$ in $0.2 \%$ Triton X-100 $(1 \mathrm{ml} / 10 \mathrm{mg})$ by a motor-driven pestle in an all-glass 
Potter-Elvehjem homogeniser. Homogenates were clarified by centrifugation at $16500 \mathrm{rev} / \mathrm{min}, 30 \mathrm{~min}$, $4^{\circ} \mathrm{C}$. Aliquots of supernatant fluid were assayed colorimetrically for acid phosphatase activity with p-nitrophenylphosphate substrate (Sigma Chemical Co., St. Louis, Mo.; Kit 104) and for beta-glucuronidase activity with phenolpthalein glucuronic acid substrate (Sigma Kit 325).

Cartilage breakdown was studied in vitro by means of the bovine nasal cartilage disc-synovial mince coculture method we have described previously. ${ }^{9}$ Briefly, uniform $1 \times 8 \mathrm{~mm}$ cartilage discs prepared from bovine nasal septum were established as organ cultures on stainless steel grids. Each culture was fed at 2-day intervals with $2 \mathrm{ml}$ Dulbecco's modified Eagle's medium (high glucose) supplemented with $10 \%(\mathrm{v} / \mathrm{v})$ fetal calf serum, $100 \mu \mathrm{g} / \mathrm{ml}$ streptomycin sulphate, $100 \mathrm{U} / \mathrm{ml}$ penicillin, $5 \mu \mathrm{g} / \mathrm{ml}$ amphotericin-B, and incubated at $37^{\circ} \mathrm{C}$ in steel chambers (Baird and Tatlock, London) flushed with $5 \% \mathrm{CO}_{2}: 95 \% \mathrm{O}_{2}$. Aliquots of minced synovium were applied to replicate cultures, overlapping the cartilage edge by a few $\mathrm{mm}$. Experiments were continued for 8 to 12 days from the time of addition of synovium. Proteoglycan released into culture medium was assayed by the alcian blue-complexing method ${ }^{17}$ with chondroitin sulphate as the standard.

\section{Results}

All the animals tolerated their injections well. In dextran sulphate injected knees there was evident heat and swelling during the first 2 weeks, which later subsided. When the animals were killed these knees were still slightly swollen and usually showed reddish brown discoloration and apparent hyperplasia of synovial lining when the joint cavity was opened. There was no obvious gross change of articular surfaces other than some loss of normal glossiness.

A total of 10 rabbits received intra-articular dextran sulphate in one knee and either saline, dextran or chondroitin sulphate in the contralateral joint. The enzyme activities of synovial homogenates are shown in Fig. 1. Data are presented as the acid phosphatase (Fig. 1a) or beta-glucuronidase (Fig. 1b) level in homogenised dextran sulphate synovium versus the activity in the contralateral control knee for each animal. If there were no significant effect of dextran sulphate, all points would fall on the $45^{\circ}$ line of equivalence. In each animal, however, the dextran sulphate side exhibited greater acid phosphatase activity than the contralateral side, irrespective of the latter agent (Fig. 1a). Similarly, in 9 of 10 instances beta-glucuronidase values also fell above the line (Fig. 1b). Analysis by the Wilcoxon test for pair differences ${ }^{18}$ was highly significant in both cases $(\mathrm{P}<0.01)$. The close correspondence between the acid phosphatase and betaglucuronidase responses is in keeping with their common lysosomal origin, ${ }^{19}$ and indeed when both enzyme values were compared in all 20 knees irrespective of the substance injected, there was a significant correlation $(\mathrm{r}=0.62, \mathrm{P}<0.01)$.

The degradative capacity of dextran sulphate treated synovia was still evident as late as the 8th

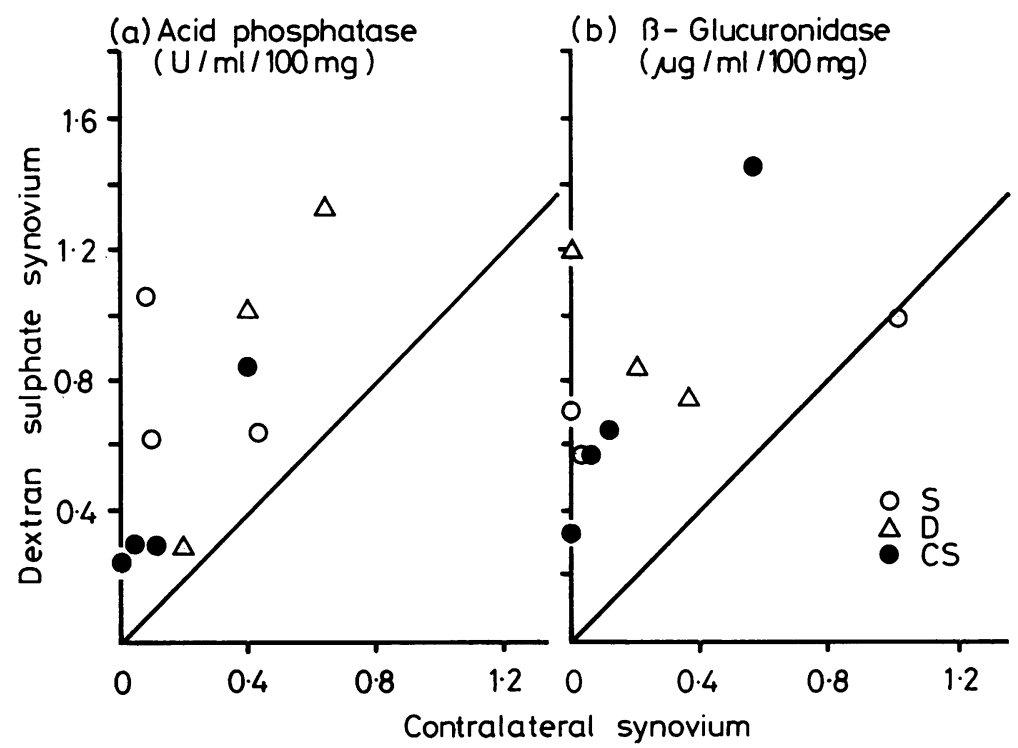

Fig. 1 Comparative effects of intra-articular dextran sulphate and contralateral substances on synovial lysosomal enzyme activities. Ten rabbits were injected with dextran sulphate in one knee, and saline ( 0 ), or dextran $(\triangle)$, or chondroitin sulphate $(\bullet)$ in the contralateral knee. Acid phosphatase (a) and beta-glucuronidase (b) activities in homogenised synovia are plotted as the dextran sulphate vs. contralateral value in each instance. The diagonals each represent a line of equivalence.

Contralateral synovium 
week after injection. The nature of the response in vitro is illustrated in the experiment shown in Fig. 2. The addition of dextran sulphate synovium (DSS) did not immediately alter the course of spontanteous proteoglycan release into culture medium, but this was significantly increased 4 days later and continued at a more rapid rate until the end of the experiment. Erosion of the cartilage disc in the area adjacent to the synovial explant was frequently evident at that time, and was similar to the gross changes we have described in experiments using human rheumatoid synovia. ${ }^{9}$ Exposure to saline treated synovium (NS) gave values slightly lower than basal, but this apparent protective effect was not statistically significant even at day $10(t=1 \cdot 72$; $P>0 \cdot 1)$.

Pooled data from a number of similar experiments

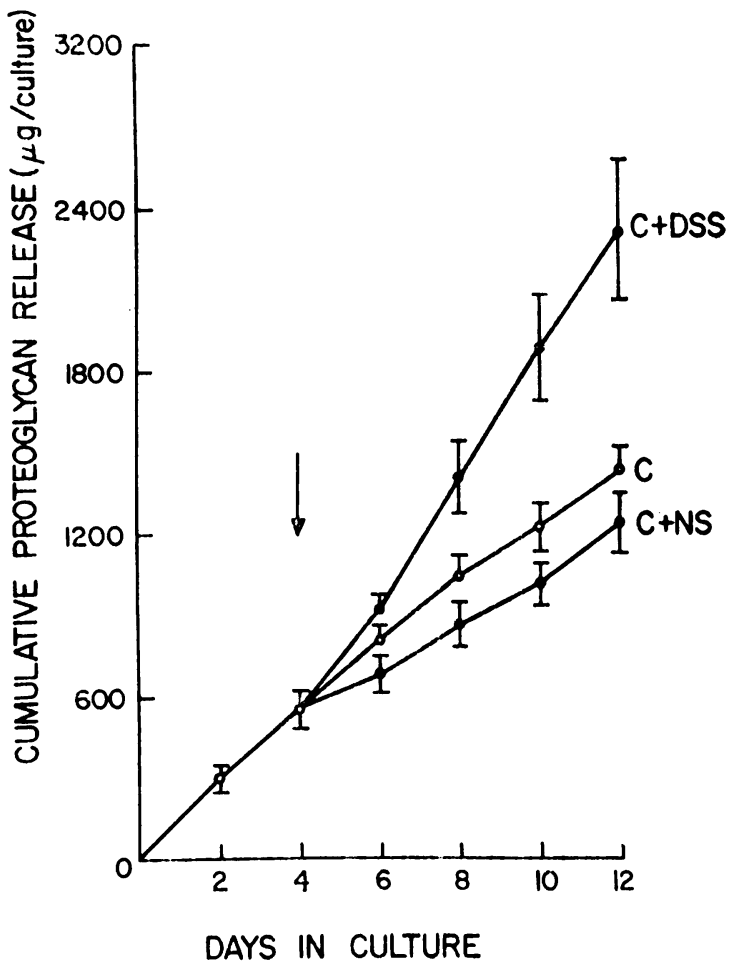

Fig. 2 Cartilage breakdown in dextran sulphate induced arthritis. Bovine nasal cartilage discs were established in organ culture on day 0 and fed by complete medium change every 2 days. Minced synovium derived from a single rabbit knee treated with dextran sulphate 8 weeks previously was added to one-third of the cultures at the arrow $(C+D S S)$. Synovium taken from the contralateral saline-injected knee was added to a second group $(C+N S)$, and the remaining onethird were carried as cartilage alone $(C)$. Values are the mean $\pm S E M$ of 6 cultures in each group. are shown in Fig. 3. In each experiment basal proteoglycan accumulation in control cultures containing cartilage discs alone was set at $1 \cdot 0$; values in synovium-containing cultures were expressed as treatment/control $(\mathrm{T} / \mathrm{C})$ ratios. No significant effect was evident during the first 4 days after addition of synovium, reflecting the lag usually noted in individual experiments. Proteoglycan releasewas abruptly stimulated between the 4th and 6th days, with an eventual doubling of medium proteoglycan content by the 10th day in complete cultures. These data are closely comparable to our previous findings using synovia from patients with rheumatoid arthritis or rabbits with antigen-induced arthritis. ${ }^{9}$

The relationship between degradative capacity in vitro and synovial lysosomal enzyme content at the time of the animals' being killed is illustrated in Table 1. In 4 animals given dextran sulphate in one knee and chondroitin sulphate in the other there was a parallelism between initial acid phosphatase or beta-glucuronidase activity and the 8-day cumulative proteoglycan release. Dextran sulphate synovia with high lysosomal enzyme content induced significantly greater proteoglycan release $(\mathrm{T} / \mathrm{C}$ ratio $=2 \cdot 04)$ than did chondroitin sulphate-

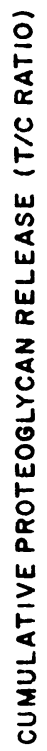

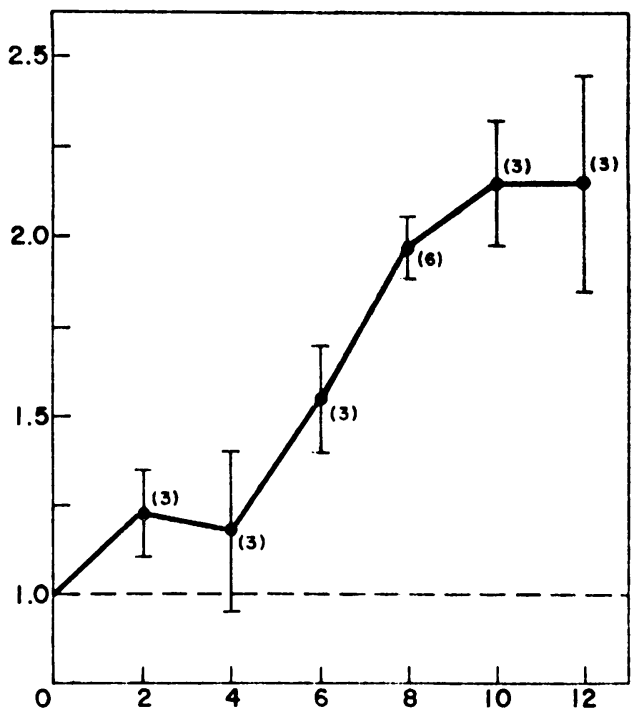

DAYS AFTER ADDITION OF SYNOVIUM

Fig. 3 Cartilage degradation in dextran sulphate induced arthritis. In each experiment basal $P G$ release from cartilage alone was set at $1 \cdot 0$, and the treatment effect of cocultivation with dextran sulphate synovium was related to this baseline. Pooled data are expressed as treatment/control $(T / C)$ ratios, and individual values means $\pm S E M$ of $(n)$ experiments. 
Table 1 Comparison of cartilage degradative capacity with initial enzyme activities of synovia from injected knees

\begin{tabular}{llll}
\hline Injection & $\begin{array}{l}\text { Acid phosphatase } \\
(U / \mathrm{ml} / 100 \mathrm{mg})\end{array}$ & $\begin{array}{l}\beta-G l u c u r o n i d a s e \\
(\mu \mathrm{g} / \mathrm{ml} / 100 \mathrm{mg})\end{array}$ & $\begin{array}{l}\text { Proteoglycan } \\
\text { release }(\mathrm{T} / \mathrm{C} \\
\text { ratio })\end{array}$ \\
\hline $\begin{array}{l}\text { Saline (8) } \\
\begin{array}{c}\text { Chondroitin } \\
\text { sulphate (4) }\end{array}\end{array}$ & $0.110 \pm 0.025$ & $0.192 \pm 0.064$ & $0.93 a$ \\
$\begin{array}{c}\text { Dextran } \\
\text { sulphate (4) }\end{array}$ & $0.411 \pm 0.143 c$ & $0.751 \pm 0.245 c$ & $2.04 \pm 0.12 d, e$ \\
\hline
\end{tabular}

Four rabbits were injected with chondroitin sulphate in one knee and dextran sulphate in the other; an additional 4 rabbits received saline. Synovia were harvested after 8 weeks. Cumulative 8-day proteoglycan release from cartilage is expressed as the treatment/control ratio of 5 cultures in each experiment as described in Fig. 3. Values are means \pm SEM of (n) synovia. (a) data from Fig. 1; (b) $\mathbf{P}<0.02$; (c) $\mathbf{P}<0.02$ vs. saline; (d) $\mathrm{P}<0.001 ;(e) \mathrm{P}<0.02$ vs. chondroitin sulphate.

synovia $(T / C$ ratio $=1 \cdot 48)$. Despite normal levels of both acid phosphatase and beta-glucuronidase activities in the latter, chondroitin sulphate-synovia were nevertheless capable of stimulating significant cartilage breakdown in vitro $(P<0.02)$. Thus, while there was an apparent parallelism between cartilage destruction in vitro and lysosomal enzyme content (in vivo), the responses could be dissociated.

\section{Discussion}

Diseases of articular cartilage, although differing in their clinical features, share certain common pathophysiological mechanisms which ultimately produce cartilage destruction. Lysosomal enzymes play a key role in the final pathway, ${ }^{1,2}$ although specifically which cells are involved and what the sequence of events may be has not been elucidated. Acid cathepsins, neutral proteases, elastase, collagenase, hyaluronidase - enzymes capable of initiating degradation of cartilage matrix-have been demonstrated in one or more of the cellular elements and synovial fluid which comprise the diarthrodial joint. $^{3}$ Subsequent degradation of large cleavage products via a number of other enzymes such as exoglycosidases and sulphatases is thought to occur either in the immediate extracellular environment or after cellular ingestion of macromolecules and formation of secondary lysosomes. ${ }^{3,20}$ The interaction of these many cellular elements, their products, and surrounding matrix occur not only from direct invasion (as by rheumatoid pannus) but may also occur at a distance. ${ }^{8,21}$ This flow of informational molecules between synovium and cartilage may be bidirectional, for cartilage does not serve merely as an inert substrate.$^{8.9}$ Moreover, the possibility that matrix proteoglycans released from their normal seclusion by initial cleavage may perpetuate the inflammatory response has led to the development of a number of in-vivo models of polysaccharideinduced arthritis. ${ }^{12,13,16,22}$

We have demonstrated that a short course of intra-articular dextran sulphate (average mol wt $5 \times 10^{5}$ ) regularly produced a persistent inflammatory arthritis in rabbits, with synovial hyperplasia, periarticular inflammation and the capacity for cartilage degradation in vitro. In contrast, injection with either uncharged dextran of the same molecular weight, or the naturally occurring glycosaminoglycan chondroitin sulphate, bearing a high charge density but of molecular weight less than $50000^{23,24} \mathrm{did}^{-}$not reproduce these gross findings. Dextran sulphate treatment increased the content of the lysosomal marker enzymes, acid phosphatase and beta-glucuronidase. This response was consistently greater than to any of the other test substances-saline, dextran, or chondroitin sulphate (Fig. 1a and b). In an independent bioassay using cartilage synovium cocultures, the degradation of cartilage in vitro was increased approximately 2-fold in the presence of dextran sulphate synovium. This paralleled the lysosomal enzyme content of synovium at the animals' death. However, it is noteworthy that the two indicators of synovial activity were not necessarily concordant. Chondroitin sulphate synovia which contained normal levels of the 2 marker enzymes nonetheless were capable of significant resorptive activity in organ culture. Possible explanations include significant increases in other unmeasured enzymes, or the unmasking in vitro of degradative potential held in abeyance in the intact animal.

The significance of negative charge versus molecular weight as the major determinant for eliciting a response remains unanswered by the present experiments. Neither high charge density nor high molecular weight alone are sufficient to explain the phenomenon, but induction of the degradative response appears to depend on some interaction of these 2 variables. Recent work from our laboratory (Aaron and Sledge, unpublished) with carrageenins of equivalent charge but differing molecular weights lends support to this hypothesis.

The authors thank Sonya Shortkroff and Pat Mark for expert technical assistance. Supported by National Institutes of Health grant numbers AM17930, AM18268, and the US Veterans Administration.

\section{References}

1 Hollander J L, McCarty D J Jr, Astorga G, CastroMurillo E. Studies on the pathogenesis of rheumatoid arthritis. Ann Intern Med 1965; 62: 271-280.

2 Weissmann G. Lysosomal mechanisms of tissue injury in arthritis. $N$ Engl J Med 1972; 286: 141-147.

3 Barrett A J. The enzymic degradation of cartilage matrix. In: Burleigh P M C, Poole A R eds. Dynamics of Con- 
nective Tissue Macromolecules. Amsterdam: NorthHolland Publishing Company 1975; 189-125.

4 Bosmann H B. Cellular control of macromolecular synthesis: rates of synthesis of extracellular macromolecules during and after depletion by papain. Proc R Soc (Biol) 1968; 169 : 399-425.

5 Jackson S F. Environmental control of macromolecular synthesis in cartilage and bone: morphogenetic response to hyaluronidase. Proc $R$ Soc Lond (Biol) 1970; 175: 405-453.

- Hardingham T E, Jackson S F, Muir H. Replacement of proteoglycans in embryonic chick cartilage in organ culture after treatment with testicular hyaluronidase. Biochem J 1972; 129: 101-112.

7 Mankin H J. Biochemical and metabolic abnormalities in osteoarthritic human cartilage. Fed Proc 1973; 32: 14781480.

${ }^{8}$ Fell $\mathbf{H ~ B}$, Jubb $\mathrm{R} \mathrm{W}$. The effect of synovial tissue on the breakdown of articular cartilage in organ culture. Arthritis Rheum 1977; 20: 1359-1371.

- Steinberg J, Sledge C B, Noble J, Stirrat C R. A tissueculture model of cartilage breakdown in rheumatoid arthritis. Quantitative aspects of proteoglycan release. Biochem J 1979; 180: 403-412.

10 Schorlemmer H U, Burger R, Hylton W, Allison A C. Induction of lysosomal enzyme release from cultured macrophages by dextran sulfate. Clin Immunol Immunopathol 1977; 7: 88-96.

11 Sledge C B, Blackburn W W. (1968). Heparin, dextran and dextran sulfate: effect on lysosomes from embryonic cartilage. Calcif Tissue Res 1968; 2 (Suppl): 65-65A.

12 Gardner D L. Production of arthritis in the rabbit by the local injection of the mucopolysaccharide carrageenin. Ann Rheum Dis 1960; 19: 369-376.

13 Lowther D A, Gillard G C. Carrageenin-induced arthritis. I. The effect of intra-articular carrageenin on the chemical composition of articular cartilage. Arthritis Rheum 1976; 19: 769-776.

14 Gillard G C, Lowther D A. Carrageenin-induced arthritis. II. Effect of intra-articular injection of carrageenin on the synthesis of proteoglycan in articular cartilage. Arthritis Rheum 1976; 19: 918-922.

15 Lowther D A, Gillard G C, Baxter E, Handley C J, Rich K A. Carrageenin induced arthritis. III. Proteolytic enzymes present in rabbit knees joints after a single intra-articular injection of carrageenin. Arthritis Rheum 1976; 19: 1287-1294.

16 Steinberg J, Gillies H, Sledge C B. Polysaccharideinduced arthritis. Clin Res 1978; 26: 625A.

17 Whiteman $P$. The quantitative measurement of alcan blue-glycosaminoglycan complexes. Biochem $J$ 1973; 131 : 343-350.

18 Documenta Geigy Scientific Tables. Ed. K Diem. Ardsley N Y: Geigy Chemical Corporation 1962.

19 DeDuve C. The lysosome in retrospect. In: Dingle J T, Fell H B, eds. Lysosomes in Biology and Pathology. Amsterdam: North-Holland Publishing Company 1969; 1: $3-40$.

20 Dingle $\mathrm{J} \mathrm{T}$, The extracellular secretion of lysosomal enzymes. In: Dingle J T, Fell B H, eds. Lysosomes in Biology and Pathology. Amsterdam: North-Holland Publishing Company 1969; 2: 421-436.

21 Mitchell N S, Shepard N. Changes in proteoglycan and collagen in cartilage in rheumatoid arthritis. J Bone Joint Surg 1978; 60A: 349-354.

22 Muirden K D, Peace G. (1969). Light and electron microscope studies in carrageenin, adjuvant, and tuberculin-induced arthritis. Ann Rheum Dis 1969; 28: 392401.

23 Wasteson A. Properties of fractional chondroitin sulphate from ox nasal septa. Biochem $J 1971$; 122 : 477-485.

24 Mathews M B. Connective Tissue-Macromolecular Structure and Evolution. New York: Springer 1975; 95. 